\title{
What do we need to make genetic biomarker-guided treatment for renal cell carcinoma a reality?
}

\author{
"Entering the era of next generation sequencing, future \\ studies including also rare genetic variants ... are an interesting \\ prospective in our search for genetic biomarkers."
}

First draft submitted: 24 October 2016; Accepted for publication: 24 October 2016; Published online: 14 December 2016

\section{Keywords: biomarker $\bullet$ pharmacogenetics $\bullet$ renal cell carcinoma $\bullet$ SNP $\bullet$ targeted therapy}

Renal cell carcinoma (RCC) is the most common type of kidney cancer, in more than $80 \%$ caused by a $V H L$ gene mutation that allows tumor growth through secretion of proangiogenic factors. Worldwide, 350,000 persons are diagnosed with RCC each year and this number is increasing. Roughly $17 \%$ of patients present with metastases at first diagnosis and have a poor prognosis. The vast majority of patients have a clear cell RCC histological subtype as opposed to nonclear cells with a worse prognosis. Surgical removal is the best option in an early stage of the disease, but may not always be possible because of a poor performance status or metastases [1].

Since 2005, the era of targeted therapy has arisen, antiangiogenic therapy has significantly improved the treatment outcome of patients with RCC. The first choice of treatment in metastatic RCC (mRCC) is systemic therapy with a tyrosine kinase inhibitor (TKI) that acts on VEGF receptors among other tyrosine kinases. Sorafenib and sunitinib were the first TKIs that showed improved patient outcomes compared with the former cytokines IL-2 and IFN- $\alpha$ [2]. The pivotal Phase III trial of sunitinib showed an improvement in progression-free survival and objective response rate $(\mathrm{p}<0.001)$ and a trend toward a better overall survival $(\mathrm{p}=0.051)$ compared with IFN- $\alpha$ [2]. The mTOR inhibitors, everolimus and temsirolimus, are given to patients with a poor prog- nosis. Bevacizumab, a VEGF inhibitor and monoclonal antibody, is commonly used in combination with an mTOR inhibitor. Later, TKIs pazopanib and axitinib were approved for mRCC treatment, and pazopanib is currently prescribed in the same extent as sunitinib [3]. New game changers in the field for second-line treatment are the TKIs lenvatinib, cabozantinib and the monoclonal antibody nivolumab. These drugs show an improved efficacy compared with everolimus. Especially, the arrival of nivolumab (US FDA approved for mRCC in October 2015) may well mean that we are facing a new era of 'targeted immunotherapy'. Nivolumab is a programmed death-1 checkpoint inhibitor that releases the brakes on $\mathrm{T}$ cells activating tumor immune surveillance [4-6].

With the expanding drug arsenal in $\mathrm{mRCC}$, each with its own drug-specific pharmacokinetics (PK) and pharmacodynamics (PD) profile, the possibility of opting for the most effective treatment for a specific individual has grown. However, despite successful advances in RCC treatment, we are not yet able to give patients an optimally effective and safe treatment without adjustments afterwards. There is a large variability in individual response to TKIs but also to nivolumab, and there is a lack of biomarkers that predict treatment outcome. Some germline pharmacogenetic markers seem promising to elucidate differences in efficacy and toxicity in patients receiving

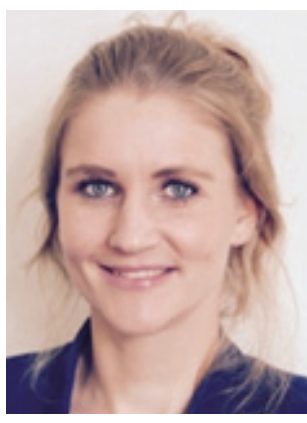

Meta H Diekstra

Department of Clinical Pharmacy \& Toxicology, Leiden University Medical Center, Leiden, The Netherlands

Xiaoyan Liu

Department of Clinical Pharmacy \& Toxicology, Leiden University Medical Center, Leiden, The Netherlands

Jesse J Swen

Department of Clinical Pharmacy \& Toxicology, Leiden University Medical Center, Leiden, The Netherlands

Henk-Jan Guchelaar Author for correspondence: Department of Clinical Pharmacy \& Toxicology, Leiden University Medical Center, Leiden, The Netherlands Tel : +310715262790 H.J.Guchelaar@lumc.nl

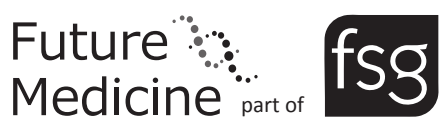


targeted therapy. Candidate gene studies interrogating SNPs located in genes related to the PK and PD of the drug of interest have been performed [7-10]. For sunitinib, the most interesting findings were SNPs in genes involved in PK encoding the CYP3A5 enzyme and the efflux transporter ATP-binding cassette ABCB1. SNPs in $C Y P 3 A 5$ and $A B C B 1$ were confirmed to be associated with sunitinib treatment outcome in a large pooled sample set of $333 \mathrm{mRCC}$ patients [9]. These results are supported by the effect of these SNPs in CYP3A5 and $A B C B 1$ on clearance of sunitinib and its active metabolite [10]. Related to PD, SNPs in VEGFR1 are associated with progression-free survival and overall survival on sunitinib, and this was validated in independent cohorts [11]. For pazopanib, SNPs in UGT1A1 and IL8 show the highest evidence for association with toxicity and survival, respectively [12-14].

\section{"Although the need for biomarkers is urgent, we should not rush on implementation without valid evidence."}

Still, the selection of SNPs in candidate gene studies is restricted to our current knowledge on PK and PD. About a decade ago, pharmacogenetic investigational methods were reinforced with the arrival of genomewide association studies. In a genome-wide association study about 1 million common genetic variants can be tested for association with drug outcomes and thereby provides an unbiased approach as compared with the candidate gene studies. Such a study has been set up for sunitinib and pazopanib as a part of the European collaborative project on EuroTARGET [15]. Entering the era of next generation sequencing, future studies including also rare genetic variants (minor allele frequency $<1 \%$ ) are an interesting prospective in our search for genetic biomarkers. Especially, because rare genetic variants in $C Y P 3 A 4$, the most important drug metabolizing enzymes, have an effect on functional variability [16].

It seems unjustified that none of the aforementioned genetic variants have been implemented into clinical practice, but several barriers keep this from happening [17]. One of the difficulties is the large heterogeneity among pharmacogenetic studies: patients have different ethnicities with corresponding allele frequencies; studies may be underpowered; and tested end points can vary in definitions and may not have been collected with the intention to perform pharmacogenetic association analyses. Changes in clinical practice owing to the experience gained in RCC treatment, such as earlier dose reductions or altered dosing schedules to prevent severe adverse events, may alter treatment outcomes. Pharmacogenetic studies investigating somatic DNA (instead of germline DNA) can be biased by intratumor heterogeneity, in other words, mutations in the tumor result in a different genetic profile and probably cause adaptive resistance to targeted treatment. The detection of ctDNA may be used to monitor dynamic changes and help individualize treatment. And what about 'negative' findings with p-values above 0.05 ? These are often not published and therefore considered as nonexisting which can blur our vision. Furthermore, findings from these studies have either not been validated in independent cohorts or are merely confirmed in small cohorts based on retrospective data. As a rule, prospective validation in an independent cohort is vital to bring SNP testing to the clinic. Yet, this has not stopped $K R A S$ mutation status to be applied as a biomarker for response to anti-EGFR treatment in colorectal cancer [17].

Although the need for biomarkers is urgent, we should not rush on implementation without valid evidence. One of the main barriers that withhold clinical use is that no consensus has been reached on a clear definition for clinical utility of pharmacogenetic testing to guide individual dosing regimens of targeted therapies. In general, clinical utility means that there is evidence that the test will improve patients' treatment outcome. But what exactly this evidence is remains unclear. In order to make progress, we first need to make sure that we have universally applicable requirements so that we can waive the debate on clinical utility. The indisputable proof to use a pharmacogenetics test should be based on a critical evaluation of available studies on the genetic variant of interest with an assessment on sample size, ethnicity, end point definitions, effect sizes, significance thresholds and the level of evidence regarding validation of findings in prospective studies. Then and there, we can concentrate on successful use of genetic markers by all healthcare professionals and facilitate ready-to-use recommendations. The Clinical Pharmacogenetics Implementation Consortium and the Dutch Pharmacogenetics Working Group already take this responsibility in providing clinical guidelines on genotyping results [18,19]. As a final point, pharmacogenetic testing is not easily accepted for uptake in clinical practice, which could be accomplished by active support of pharmaceutical companies and patient groups [17].

In the meantime, while we are not yet able to predict, we are able to adjust. Therapeutic drug monitoring (TDM) provides an accurate and feasible option to guide treatment with targeted therapies in mRCC using measured drug concentrations in blood to determine PK/PD parameters, and adjust the dose if needed. Several criteria need to be fulfilled to apply TDM; the drug has a narrow therapeutic window; 
there is a relation between drug concentration and effect; there is a high interindividual variability in PK; the dose cannot be optimized by clinical observation; there is no clear relation between dose and effect; and the intrapatient variability is less than the interpatient variability $[10,20]$. In addition to TDM, ongoing studies are aimed at finding a synergistic antitumor effect within the current drug arsenal against mRCC. New drug combinations or sequences targeting both the angiogenic and immunogenic characters of the tumor possibly improve patient outcomes.

In short, we know what hurdles need to be overcome before genetic biomarker-guided treatment becomes a reality. We need to provide scientific evidence, demonstrate the clinical relevance, develop guidelines with recommendations to direct clinical use of pharmacogenetic testing and then need to make sure that the use of pharmacogenetic tests is widely accepted [17-19]. Efforts for broad testing of genetic variants and vali-

\section{References}

Papers of special note have been highlighted as:

- of interest; $\bullet$ of considerable interest

1 Capitanio U, Montorsi F Renal cancer. Lancet 387(10021), 894-906 (2016).

2 Motzer RJ, Hutson TE, Tomczak P et al. Overall survival and updated results for sunitinib compared with interferon alfa in patients with metastatic renal cell carcinoma. J. Clin. Oncol. 27(22), 3584-3590 (2009).

- In the pivotal Phase III trial, sunitinib shows an improved efficacy compared with the former standard therapy IFN- $\alpha$.

3 Motzer RJ, Hutson TE, Cella D et al. Pazopanib versus sunitinib in metastatic renal-cell carcinoma. N. Engl. J. Med. 369(8), 722-731 (2013).

4 Glen H. Lenvatinib therapy for the treatment of patients with advanced renal cell carcinoma. Future Oncol. 12(19), 2195-2204 (2016).

5 Choueiri TK, Escudier B, Powles T et al. Cabozantinib versus everolimus in advanced renal cell carcinoma (METEOR): final results from a randomised, open-label, Phase III trial. Lancet Oncol. 17(7), 917-927 (2016).

6 Motzer RJ, Escudier B, McDermott DF et al. Nivolumab versus everolimus in advanced renal-cell carcinoma. N. Engl. J. Med. 373(19), 1803-1813 (2015).

-• The programmed death-1 checkpoint inhibitor nivolumab shows improved survival outcomes compared with everolimus and is currently used as a second-line treatment in metastatic renal cell carcinoma.

7 van Erp NP, Eechoute K, van der Veldt AA et al. Pharmacogenetic pathway analysis for determination of sunitinib-induced toxicity. J. Clin. Oncol. 27, 4406-4412 (2009).

- The first publication of a pharmacogenetic study on sunitinib showed significantly associated SNPs with adverse events on sunitinib. dation studies have already been established. We have high expectations of the results on genetic markers for TKI treatment and the development of new successful drugs or drug combinations. Not only will we move forward in pharmacogenetic research and our endeavor to find genetic markers, these novel markers will also help brighten up the treatment of RCC patients by using a more personalized approach.

\section{Financial \& competing interests disclosure}

The research leading to these results has received funding from the European Union's Seventh Framework Programme (FP7/2007-2013) under grant agreement no 259939. The authors have no other relevant affiliations or financial involvement with any organization or entity with a financial interest in or financial conflict with the subject matter or materials discussed in the manuscript apart from those disclosed.

No writing assistance was utilized in the production of this manuscript.

8 Garcia-Donas J, Esteban E, Leandro-García LJ et al. Single nucleotide polymorphism associations with response and toxic effects in patients with advanced renal-cell carcinoma treated with first-line sunitinib: a multicentre, observational, prospective study. Lancet Oncol. 12(12), 1143-1150 (2011).

9 Diekstra M, Swen JJ, Boven E et al. CYP3A5 and ABCB1 polymorphisms as predictors for sunitinib outcome in metastatic renal cell carcinoma. Eur. Urol. 68(4), 621-629 (2015).

-. Confirms 2 out of 22 previously associated SNPs to be associated with progression-free survival and the need for dose reductions on sunitinib treatment.

10 Diekstra MH, Klümpen HJ, Lolkema MP et al. Association analysis of genetic polymorphisms in genes related to sunitinib pharmacokinetics, specifically clearance of sunitinib and SU12662. Clin. Pharmacol. Ther. 96(1), 81-89 (2014).

11 Dornbusch J, Walter M, Gottschalk A et al. Evaluation of polymorphisms in angiogenesis-related genes as predictive and prognostic markers for sunitinib-treated metastatic renal cell carcinoma patients. J. Cancer Res. Clin. Oncol. 142(6), 1171-1182 (2016).

12 Xu CF, Bing NX, Ball HA et al. Pazopanib efficacy in renal cell carcinoma: evidence for predictive genetic markers in angiogenesis-related and exposure-related genes. J. Clin. Oncol. 29(18), 2557-2564 (2011).

- The first publication of a pharmacogenetic study on pazopanib showed significantly associated SNPs with response to pazopanib.

13 Motzer RJ, Johnson T, Choueiri TK et al. Hyperbilirubinemia in pazopanib- or sunitinib-treated patients in COMPARZ is associated with UGT1A1 polymorphisms. Ann. Oncol. 24(11), 2927-2928 (2013).

14 Johnson T, Xu CF, Choueiri TK et al. Genome-wide association study (GWAS) of efficacy and safety endpoints in pazopanib- or sunitinib-treated patients with renal cell 
carcinoma (RCC). Presented at: 50th Annual Meeting of American Society Of Clinical Oncology (ASCO). Chicago, IL, USA, 30 May-3 June 2014.

15 EuroTARGET: TArgeted therapy in Renal cell cancer: GEnetic and Tumour related biomarkers for response and toxicity.

www.eurotargetproject.eu

16 Kozyra M, Ingelman-Sundberg M, Lauschke VM. Rare genetic variants in cellular transporters, metabolic enzymes, and nuclear receptors can be important determinants of interindividual differences in drug response. Genet. Med. doi:10.1038/gim.2016.33 (2016) (Epub ahead of print).

17 Swen JJ, Huizinga TW, Gelderblom $\mathrm{H}$ et al. Translating pharmacogenomics: challenges on the road to the clinic. PLoS. Med. 4(8), 1317-1324 (2007).
- Describes the most important barriers for clinical implementation of pharmacogenomic testing.

18 Swen JJ, Nijenhuis M, de Boer A et al. Pharmacogenetics: from bench to byte - an update of guidelines. Clin. Pharmacol. Ther. 89(5), 662-673 (2011).

19 Guchelaar HJ, Gelderblom H, van der Straaten T et al. Pharmacogenetics in the cancer clinic: from candidate gene studies to next-generation sequencing. Clin. Pharmacol. Ther. 95(4), 383-385 (2014).

20 Yu H, Steeghs N, Nijenhuis CM et al. Practical guidelines for therapeutic drug monitoring of anticancer tyrosine kinase inhibitors: focus on the pharmacokinetic targets. Clin. Pharmacokinet. 53(4), 305-325 (2014). 